\title{
ESTUDO EPIDEMIOLÓGICO DE DIFTERIA NA REGIĀO DA GRANDE SÃO PAULO, 1969
}

\author{
Marcilia de Araujo Medrado FARIA (1) \\ Claudio Luiz LUCARELLI (2) \\ Claudelina Maria PIRES (2) \\ Clelgen Luiz BONETTI (2) \\ Cristina MISHIKAWARA (2) \\ Daniel Massakasu KATAYAMA (2) \\ Edison Joshi NAKAGAWA (2)
}

\begin{abstract}
Faria, M. de A. M. et al. - Estudo epidemiológico de difteria na região da Grande São Paulo, 1969. Rev. Saúde públ., S. Paulo, 5:213-20, 1971.

Resumo - Realizou-se um levantamento epidemiológico de Difteria, utilizando-se uma amostra de 29 pacientes (GRUPO POSITIVO), a qual correspondeu a $13,5 \%$ de crianças internadas no Hospital de Isolamento Emílio Ribas, . São Paulo, SP., entre fevereiro a outubro de 1969. Através de pareamento por idade e sexo foram selecionados, entre os vizinhos dos pacientes, 24 crianças (GRUPO CONTROLE). A maioria dos pacientes provinha de bairros ou municípios operários da Grande São Paulo, vivendo sob precárias condições educacionais de saneamento e higiene. Através da história pregressa de vacinação, observou-se que nos 2 grupos é elevado o número de crianças não vacinadas com as três doses iniciais da triplice. Porém, no Grupo Positivo, êste fato é observado em $100 \%$ das crianças. Não houve diferenças significativas em relação ao teste de Schick realizado entre os irmãos dos indivíduos dos dois grupos. Entretanto, as frequiências de Schick negativo foram $61,5 \%$ e $75,5 \%$, respectivamente, para os Grupos Contrôle e Positivo. Estes dados indicam que os programas de vacinação não têm conseguido resultados satisfatórios na imunização das populaçóes de baixo nível econômico-social.
\end{abstract} Schick.

Unitermos - Difteria (epidemiologia) *; Vacinação tríplice *; Teste de

\section{N T R O D U CAO}

A incidência da difteria tem diminuído acentuadamente nos paises desenvolvidos e, em alguns dêles, a doença está pràticamente sob contrôl's". Nos países subdesenvolvidos, embora se tenha observado apreciável redução dos índices de morbidade e mortalidade, os mesmos continuam bastante elevados ${ }^{2}$. E o que se verifica no Município de São Paulo onde ainda se encontra elevada incidência anual ${ }^{(3)}$. Em 1969 , aproximadamente $1,4 \%$ das mortes ocorridas, de 1 a 4 anos, foram devidas a esta doença ${ }^{1}$.

Recebido para publicação em 26-7-1971.

(1) Do Departamento de Medicina Preventiva da Faculdade de Medicina da USP - Av. Dr. Arnaldo, 455 - Sāo Paulo, SP, Brasil.

(2) Alunos do 2.0 ano da Faculdade de Medicina da USP

(3) Informacões fornecidas pelo Hospital de Isolamento "Emilio Ribas" — São Paulo, SP. 
FARIA, M. de A. M. et al. - Estudo epldemiológico de difteria na regiăo da Grande São Paulo, 1969. Rev. Saride públ., S. Paulo, 5:213-20, 1971.

Numa tentativa de avaliação dos fatôres pré-patogênicos, responsáveis pela manutençâa dos elevados índices da doença, realizamos um levantamento epidemiológico, a partir de pacientes diftéricos internados no Hospital de Isolamento Emílio Ribas, São Paulo, SP (H.I.E.R.). Foi da. do enfoque especial às condições de vida e gráus de susceptibilidade à infecção, nos grupos familiares destas crianças diftéricas.

\section{MATERIAL E METODOS}

A partir dos doentes internados no H.I.E.R., com diagnóstico comprovado de difteria entre os meses de fevereiro a outubro de 1969, foram selecionados casos em que não houvesse ocorrido o êxito letal. Excluindo os pacientes em que não se conseguiu identificar o local da residência e outros que haviam mudado de domicílio, foram levantados dados epidemiológicos de 29 pacientes (Grupo Positivo), o que correspondeu a uma amostra de $13,5 \%$ do total de casos (214) diagnosticados no período estudado. Para comparar as diversas variáveis, que são importantes no trabalho, foram selecionadas, entre os vizinhos dos pacientes, 24 crianças sem difteria as quais denominamos de Grupo Contrôle. O critério de escolha dêste grupo foi o pareamento com os indivíduos do Grupo Positivo, levando-se em consideração idade e sexo.

O levantamento epidemiológico consistiu no preenchimento de um questionário elaborado com o fim de obter os seguintes dados sôbre família: composição por sexo e idade, estrutura, condições sócio-econômicas e sôbre condiçōes de saneamento da residência, mecanismos de transmissão da doença e história anterior de vacinação. Foram considerados casos de vacinação completa aquêles em que as crianças haviam feito uso das 3 doses iniciais da vacina anti-diftérica. Foi realizado o teste Schick ${ }^{5}$ que consistiu na aplicação da injeção de $0,1 \mathrm{ml}$ de toxina diftérica diluída na face de flexão do antebraço esquerdo dos indivíduos seleciona- dos e respectivos familiares. Administrouse de modo semelhante 0,1 da toxina inativada (teste Malooney) no antebraço direito. Por razões de ordem prática e como se dispunha de um grupo testemunho, foi realizada uma única leitura, aproximadamente após 72 horas, da administração da toxina. O teste de Schick foi considerado positivo quando os individuos apresentavam uma área de eritema no braço esquerdo, igual ou superior a $1,5 \mathrm{~mm}$.

\section{RESUL T A DOS}

Metade dos pacientes internados no periodo mencionado, provinham do município de São Paulo, em sua maioria de bairros periféricos. Os restantes residiam em municípios da Grande São Paulo. A procedência dos 29 pacientes selecionados era semelhante à da população internada. Apenas 2 pacientes residiam em bairros centrais sendo que os demais provinham de bairros operários e periféricos. (ver Figura).

Não houve diferença entre o grau de educação e ocupação dos pais, nos 2 grupos. Em tôrno de $65 \%$, eram operários não especializados, serventes, vigias e comerciários. Embora haja diferenças percentuais do nível de educação das mães dos indivíduos do Grupo Positivo e do Grupo Contrôle $(41,4 \%$ e $26 \%$ de mães analfabetas), estas não foram significantes. A distribuição da renda familiar mensal era igual nos 2 grupos sendo que cêrca de $70 \%$ dos casos recebiam menos de dois salários mínimos. Também não se observou diferença entre os 2 grupos em relação aos dados de saneamento.

Não foi possível detectar contatos recentes que se pudessem considerar como fontes de infecção. Entretanto, houve maior contato com pessoas que tinham tido difteria no ambiente familiar do que na escola ou vizinhança.

Quando ocorreu mais de um caso de difteria no mesmo agrupamento familiar, o que correspondeu a $24,3 \%$ das famílias de pacientes, pôde-se observar que seus 


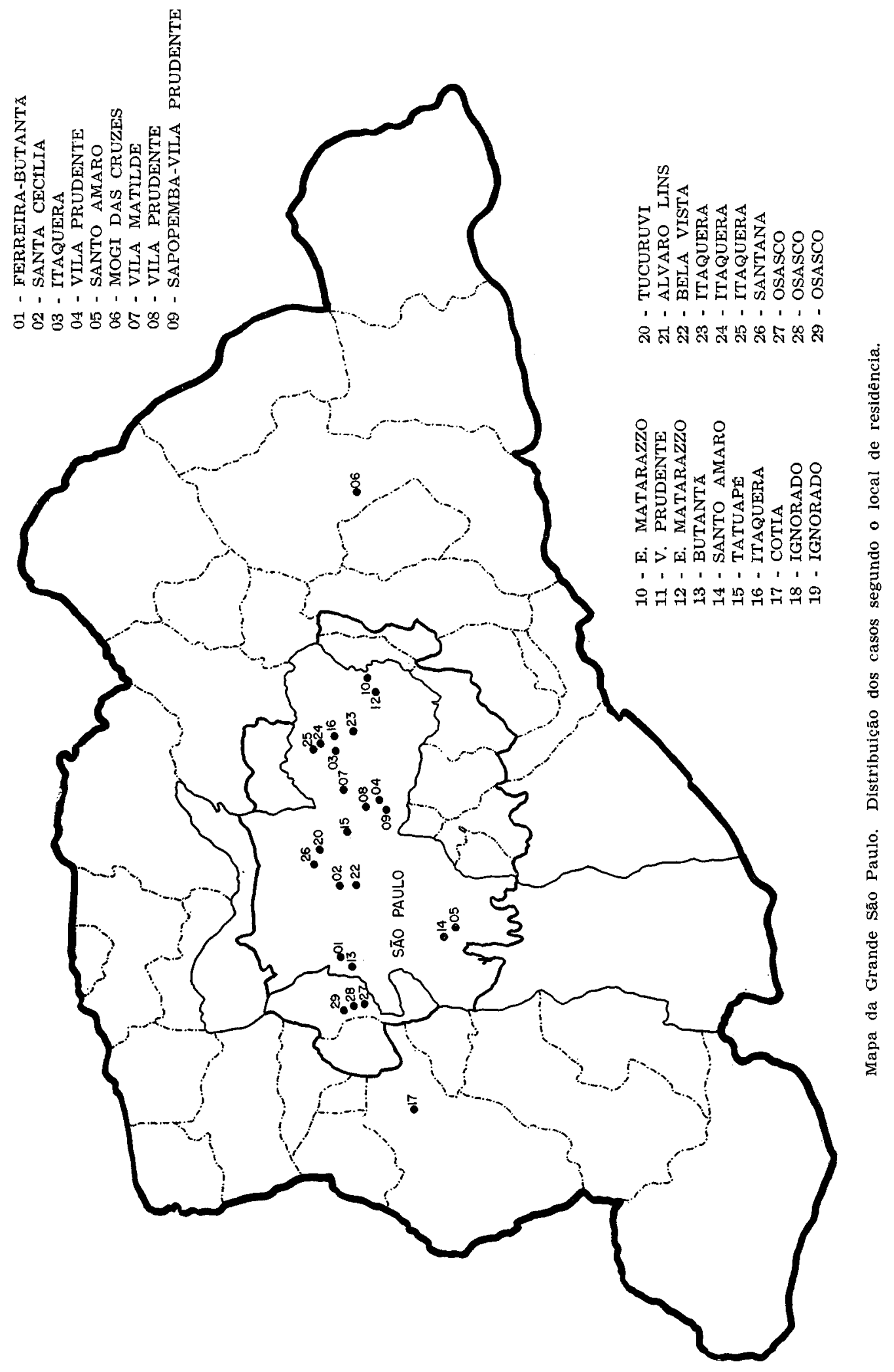


FARIA, M. de A. M. et al. - Estudo epidemiológico de difteria na região da Grande São Paulo, 1969. Rev. Sañde puibl., S. Paulo, 5:213-20, 1971.

TABELA 1

Distribuicão etária dos pacientes do Grupo Positivo e Contrôle.

\begin{tabular}{|c|c|c|c|c|c|c|c|c|}
\hline & \multirow{2}{*}{\multicolumn{2}{|c|}{ Idade }} & \multicolumn{2}{|c|}{ Grupo Positivo } & \multicolumn{2}{|c|}{ Grupo Cantrôle } & \multicolumn{2}{|c|}{ Total } \\
\hline & & & N.० & $\%$ & N.० & $\%$ & N.o & $\%$ \\
\hline & & $>2$ & 5 & $(17,2)$ & 3 & $(12,5)$ & 8 & $(15,0)$ \\
\hline & 2 & $>4$ & 7 & $(24,1)$ & 6 & $(25,0)$ & 13 & $(24,5)$ \\
\hline & $4:$ & $>6$ & $\mathbf{9}$ & $(31,0)$ & 6 & $(25,0)$ & 15 & $(28,3)$ \\
\hline & & $>8$ & 2 & $(6,9)$ & 3 & $(12,5)$ & 5 & $(9,4)$ \\
\hline & & $>10$ & 3 & $(10,3)$ & 2 & $(8,3)$ & 5 & $(9,4)$ \\
\hline & 10 & $>12$ & 2 & $(6,9)$ & 2 & $(8,3)$ & 4 & $(7,5)$ \\
\hline & 12 & $\div$ & 1 & ( 3.5$)$ & 2 & $(8.3)$ & 3 & $(5,7)$ \\
\hline Total & & & 29 & $(100,0)$ & 24 & $(100,0)$ & $\mathbf{5 3}$ & $(100,0)$ \\
\hline
\end{tabular}

TABELA 2

Distribuicāo do Grupo Positivo e do Grupo Contrôle segundo história de vacinação anterior.

\begin{tabular}{|c|c|c|c|c|c|c|}
\hline \multirow{3}{*}{ HIstória de vacinacăo } & \multicolumn{6}{|c|}{ Diagnóstico } \\
\hline & \multicolumn{2}{|c|}{ Grupo positivo } & \multicolumn{2}{|c|}{ Grupo contrôle } & \multicolumn{2}{|c|}{ Total } \\
\hline & N.० & $\%$ & N.a & $\%$ & N.o & $\%$ \\
\hline Năo vacinados & 24 & $(39,0)$ & 8 & $(53,3)$ & 32 & $(76,2)$ \\
\hline Vacinados com $1 .^{\mathrm{a}}$ dose & 3 & $(11,0)$ & 2 & $(13,3)$ & 5 & $(11,9)$ \\
\hline Vacinados com 2 doses & - & - & 1 & $(6,6)$ & 1 & $(2,4)$ \\
\hline Vacinados com 3 doses & - & - & 4 & $(26,6)$ & 4 & $(9,5)$ \\
\hline Potal (*) & 27 & $(100,0)$ & 15 & $(100,0)$ & 42 & $(100,0)$ \\
\hline
\end{tabular}

(*) A não coincidência cos totais da tabela com os de individuos estudados deve-se ao fato de que parte dêles não soube informar a histórla pregressa de vacinação. 
FARIA, M. de A. M. et al. - Estudo epidemiológico de difteria na região da Grande São Paulo, 1969. Rev. Saude puibl., S. Paulo, 5:213-20, 1971.

membros dormiam no mesmo quarto. Além disso, em 3 dos casos, dormiam no mesmo leito e usavam os mesmos utensílios e objetos pessoais.

Em relação ao tipo de assistência médica inicialmente procurada, observou-se que metade dos pacientes recorreram ao Posto Médico, 27,3\% ao médico particular e $18,2 \%$ ao farmacêutico. $\mathrm{Na}$ maioria dos casos nenhuma medida de contrôle da enfermidade foi adotada pelos serviços responsáveis; em 4 casos foi feita a vacinação dos familiares, sendo que em um caso o próprio paciente foi vacinado.

A distribuição etária dos pacientes da nossa amostra coincidiu com a mesma distribuição para a totalidade dos casos de difteria hospitalizados no período. Cêrca de $80 \%$ dos pacientes estavam na faixa etária de 1 a 10 anos. A mediana de idade dos pacientes do Grupo Positivo foi de aproximadamente 4 anos e era igual ao do Grupo Contrôle. (Tabela 1).

História prévia de vacinação - As informações obtidas de vacinações anteriores mostram que ocorreu a doença sòmente em crianças não vacinadas $(89,0 \%)$ ou quando se fêz uso de uma única dose da vacina $(11,0 \%)$. No entanto, no Grupo Contrôle, cêrca de $47,0 \%$ dos indivíduos tinham sido vacinados e $26,7 \%$ tinham seguido um esquema inicial correto de vacinação, isto é, usado as 3 doses. (Tabela 2).

Através dos Testes $\mathrm{X}^{2}$ observou-se que estas diferenças são estatìsticamente significantes. Observou-se, também, que os resultados de história prévia de vacinação dos irmãos e de grupo familiar (todos elementos da família excluíndo os casos selecionados) mostram diferenças significativas entre os dois grupos quanto à vacinação realizada anteriormente. Sòmente $10,1 \%$ dos irmãos das crianças que tiveram difteria havia feito uso da vacinação completa, enquanto que nos irmãos das crianças do grupo contrôle foi assinalada uma freqüência de $31,4 \%$.

$\mathrm{Na}$ Tabela 3 encontra-se a distribuição dos irmãos dos indivíduos dos dois grupos segundo a história de vacinação tríplice prévia.

TABELA 3

História anterior de vacinaçao dos irmãos dos indivíduos dos grupos Positivo e Contrôle.

\begin{tabular}{|c|c|c|c|c|c|c|}
\hline \multirow{2}{*}{ História de vacinação } & \multicolumn{2}{|c|}{ Grupo Positivo } & \multicolumn{2}{|c|}{ Grupo Contrôle } & \multicolumn{2}{|c|}{ Total } \\
\hline & N. $0^{\circ}$ & $\%$ & N.0 & $\%$ & N.: & $\%$ \\
\hline Năo vacinados & 63 & $(63,6)$ & 54 & $(62,8)$ & 117 & $(63,2)$ \\
\hline Vacinados com 1.a dose & 15 & $(15,2)$ & 4 & $(4,7)$ & 19 & $(10,3)$ \\
\hline Vacinados com 2.a dose & 11 & $(11,1)$ & 1 & $(1,7)$ & 12 & $(6,5)$ \\
\hline Vacinados com 3.2 dose & 10 & $(10,1)$ & 27 & $(31,4)$ & 37 & $(20,0)$ \\
\hline Total & 99 & $(100,0)$ & 86 & $(100,0)$ & 185 & $(100,0)$ \\
\hline
\end{tabular}


FARIA, M. de A. M. et al. - Estudo epidemíológico de difteria na região da Grande São Paulo, 1969. Rev. Saúde públ., S. Paulo, 5:213-20, 1971.

Teste de Schick - Foram realizadas 260 reaçōes de Schick, sendo 128 nas famílias do Grupo Contrôle e 132 nos familiares dos pacientes diftéricos. Nos pacientes que apresentaram a doença foram observados 3 indivíduos com reação de Schick positiva. A frequiência no Grupo Positivo foi de $13,0 \%$ enquanto que correspondeu a $26,0 \%$ no grupo contrôle. Não foi demonstrada através do Teste $\mathrm{X}^{2}$ significância das diferenças encontradas (Tabela 4).
Embora a freqüência de reações positivas tenha sido maior nos irmãos dos pacientes diftéricos $(24,3 \%)$ e menor nos irmãos dos indivíduos aparentemente sadios $(18,5 \%)$, o teste $\mathrm{X}^{2}$, ao nível de $5 \%$ de significância, não demonstrou diferenças quando comparados êstes resultados. $\mathrm{Na}$ Tabela 5 encontram-se os resultados do teste de Schick nos irmãos dos indivíduos dos dois grupos.

TABELA 4

Resultado do teste de Schick nos pacientes do Grupo Positivo e Grupo Contróle.

\begin{tabular}{|c|c|c|c|c|c|c|}
\hline \multirow{2}{*}{ Teste de Schick } & \multicolumn{2}{|c|}{ Grupo Positivo } & \multicolumn{2}{|c|}{ Grupo Contrôle } & \multicolumn{2}{|c|}{ Total } \\
\hline & N.o & $\%$ & N.o & $\%$ & N.o & $\%$ \\
\hline Schick + & 3 & $(13,3)$ & 6 & $(26,0)$ & 9 & $(19,6)$ \\
\hline Schick - & 20 & $(86,7)$ & 17 & $(74,0)$ & 37 & $(80,4)$ \\
\hline Total & 23 & $(100,0)$ & 23 & $(100,0)$ & 46 & $(100,0)$ \\
\hline
\end{tabular}

TABELA 5

Resultado do teste de Schick dos irmãos dos individuos dos Grupos Positivo e Controle.

\begin{tabular}{|c|c|c|c|c|c|c|}
\hline \multirow{2}{*}{ Teste de Schick } & \multicolumn{2}{|c|}{ Grupo Positivo } & \multicolumn{2}{|c|}{ Grupo Contrôle } & \multicolumn{2}{|c|}{ Total } \\
\hline & $\mathbf{N} . \mathbf{o}$ & $\%$ & N.o & $\%$ & N..$^{\circ}$ & $\%$ \\
\hline Schick + & 18 & $(24,3)$ & 12 & $(18,5)$ & $\mathbf{3 0}$ & $(21,6)$ \\
\hline Schick - & 56 & $(75,7)$ & 53 & $(81,5)$ & 109 & $(78,4)$ \\
\hline Total & 74 & $(100,0)$ & 65 & $(100,0)$ & 139 & $(100,0)$ \\
\hline
\end{tabular}

$\mathrm{X}^{2}=0,7 \quad \mathrm{P}>0,05$ 
FARIA, M. de A. M. et al. - Estudo epidemiológico de difteria na região da Grande såo Paulo, 1969. Rev. Sauide pribl., S. Paulo, 5:213-20, 1971.

Também não houve diferença dos resultados do Teste de Schick em relação a todos membros da família dos dois grupos.

\section{DISCUSSAO E CONCLUSOES}

A elevada freqüência de casos de difteria ocorridos em populações de bairros e municípios periféricos da Grande São Paulo, onde a maioria da população corresponde a operários não qualificados, com pequeno poder aquisitivo, vivendo sob precárias condições de higiene, está a indicar a estreita relação entre a prevalência da difteria e as condiçôes sócio-econômicas.

Embora não haja diferenças estatìsticamente significantes na comparação dos dados sócio-econômicos destaca-se, em têrmos percentuais, a maior freqüência de mães analfabetas e o menor tempo de residência na moradia atual. As condições econômicas e sociais condicionando entre vários outros problemas, um elevado número de indivíduos com baixa renda per capita $\mathrm{e}$ baixo nível educacional, representam o principal fator determinante da elevada frequiência $(89,0 \%)$ de criança não vacinadas no Grupo Positivo.

A baixa percentagem de vacinação com 3 doses iniciais revelada, quer pela freqüência de apenas $25,0 \%$ de irmãos dos pacientes, vacinados, quer pela freqüência de $26,7 \%$ de crianças vacinadas com idade média de 4 anos (GRUPO CONTROLE), mostra que nestas populações é enorme o número de crianças não vacinadas corretamente e portanto suscep. tíveis de adquirirem difteria. Os resultados do teste de Schick, de certo modo, reforçam esta conclusão pois, notou-se a tendência a uma maior freqüência de Schick negativa $(81,5 \%)$ nos irmãos dos indivíduos do Grupo Contrôle, em relação ao percentual observado nos irmãos das crianças que tiveram difteria $(75,5 \%)$, o que indica a maior susceptibilidade dêste grupo à doença. Por outro lado, é importante assinalar que a imunização arti- ficial ativa realizada de modo adequado com as três doses, leva à negativação da reação de Schick em cêrca de $90 \%$ da população ${ }^{4}$.

Estes dados indicam que os programas de vacinação não têm conseguido dar cobertura imunológica às crianças das populaçōes de menor poder aquisitivo e portanto mais necessitadas. Parece-nos não haver nenhuma programação sistemática eficiente, posta em execução pelos serviços públicos, que vise prevenir a doença, detectar e controlar os comunicantes. Nêste último caso, é provável que quando isto ocorre, seja feito de modo inadequado.

A não identificação da fonte de infecção pode ser atribuída à menor importância, na transmissão da doença, de contato com indivíduos doentes. Estes dados nos levam a acreditar que tenha um papel de destaque na cadeia epidemiológica da difteria, o portador sádio, o qual não pudemos detectar através dêste trabalho.

F ARTA, M. de A. M. et al. - [Epidemiological investigation of diphtheria in the area of the Great São Paulo, Brazil, 1969]. Rev. Saúde públ., S. Paulo, 5:213-20, 1971.

Summary - In the period between February to October 1969, an investigation on some characteristics of patients with diphtheria and their families in the area of the Great S. Paulo (Brazil), was accomplished. A group of 29 patients, corresponding to $13.5 \%$ of the total number of patients with the disease, admitted to the Hospital Emilio Ribas during the period, was compared with a matched control group of 24 neighbourhood's children. Most of the patients came from the fringe area of the Great S. Paulo and their families and residential quarters were characterized by precarious social, educational and hygienic conditions. The number of children with previous history of complete vaccination (three primary doses of toxoid) was strikingly low in both groups. As expected, none of the children in the case group had completed the three doses immunization schedule. Although the frequencies of negative Schick tests were $81.5 \%$ and $75.5 \%$ in the control and case groups, respectively. No significant difference was demonstrated 
FARIA, M. de A. M. et al. - Estudo epidemiológico de difteria na regiāo da Grande São Paulo, 1969. Rev. Saúde públ., S. Paulo, 5:213-20, 1971.

in the Schick reaction when comparing sibilings of children in the case group with those in the control families. The failure of the vaccination program with regard to this socio-economic group was clearly evident.

UNITERMS - Diphtheria (epidemiology)*; Triple vaccination *; Schick test.

\section{REFERENCIAS BIBLIOGRAFICAS}

1. LAURENTI, R. - Causas de mortalidade infantil em crianças de um a quatro anos em São Paulo. Rev. Saúde públ., S. Paulo, 4:101-4, 1970.
2. ORGANIZACAO PANAMERICANA DA SAODE. - Casos notificados de enfermedades de declaración obligatoria de las Americas. Washington, DC, 1969.

3. RASKA, K. - La vigilancia epidemiologica y su papel en los programas de imunizaclón. Bol. Ofic, sanit. panamer, 66:45161, 1969.

4. SARTWELL, P. E.; MAXCY, K. F. \& ROSENAU, M. J. - Medicina preventiva $e$ saúde puiblica. Lisboa, Calouste-Gulbenkian, 1969.

5. SCHICK, S. - Die Dphtherietoxen - Hantreaktion des neuschen als Verprobe der prophylaktischen. Müchen. Med. Wchnischr., 60:2608, 1913. 